\title{
Massively parallel pyrosequencing-based transcriptome analyses of small brown planthopper (Laodelphax striatellus), a vector insect transmitting rice stripe virus (RSV)
}

\author{
Fujie Zhang1,2,3, Hongyan Guo 1,2,3, Huajun Zheng ${ }^{4}$, Tong Zhou ${ }^{5}$, Yijun Zhou ${ }^{5}$, Shengyue Wang4, Rongxiang Fang 1,2, \\ Wei Qian ${ }^{* 1,2}$ and Xiaoying Chen*1,2
}

\begin{abstract}
Background: The small brown planthopper (Laodelphax striatellus) is an important agricultural pest that not only damages rice plants by sap-sucking, but also acts as a vector that transmits rice stripe virus (RSV), which can cause even more serious yield loss. Despite being a model organism for studying entomology, population biology, plant protection, molecular interactions among plants, viruses and insects, only a few genomic sequences are available for this species. To investigate its transcriptome and determine the differences between viruliferous and naïve L. striatellus, we employed 454-FLX high-throughput pyrosequencing to generate EST databases of this insect.

Results: We obtained 201,281 and 218,681 high-quality reads from viruliferous and naïve L. striatellus, respectively, with an average read length as $230 \mathrm{bp}$. These reads were assembled into contigs and two EST databases were generated. When all reads were combined, 16,885 contigs and 24,607 singletons (a total of 41,492 unigenes) were obtained, which represents a transcriptome of the insect. BlastX search against the NCBI-NR database revealed that only 6,873 (16.6\%) of these unigenes have significant matches. Comparison of the distribution of GO classification among viruliferous, naïve, and combined EST databases indicated that these libraries are broadly representative of the L. striatellus transcriptomes. Functionally diverse transcripts from RSV, endosymbiotic bacteria Wolbachia and yeast-like symbiotes were identified, which reflects the possible lifestyles of these microbial symbionts that live in the cells of the host insect. Comparative genomic analysis revealed that L. striatellus encodes similar innate immunity regulatory systems as other insects, such as RNA interference, JAK/STAT and partial Imd cascades, which might be involved in defense against viral infection. In addition, we determined the differences in gene expression between vector and naïve samples, which generated a list of candidate genes that are potentially involved in the symbiosis of L. striatellus and RSV.
\end{abstract}

Conclusions: To our knowledge, the present study is the first description of a genomic project for L. striatellus. The identification of transcripts from RSV, Wolbachia, yeast-like symbiotes and genes abundantly expressed in viruliferous insect, provided a starting-point for investigating the molecular basis of symbiosis among these organisms.

\section{Background}

Most insects act as vectors for the transmission of viruses and are thus one of the most important factors in the

* Correspondence: qianw@im.ac.cn, chenxy@im.ac.cn¹

State Key Laboratory of Plant Genomics, Institute of Microbiology, Chinese Academy of Sciences, Beijing 100101, China

1 State Key Laboratory of Plant Genomics, Institute of Microbiology, Chinese Academy of Sciences, Beijing 100101, China

Full list of author information is available at the end of the article study of the epidemiology and molecular pathology of plant or animal virus diseases. In the plant kingdom, it has been estimated that as much as $76 \%$ of viral diseases are transmitted by insects [1,2]. As the insects feed, insect-borne viruses easily penetrate the impermeable cuticle that covers the plant epidermis and are directly delivered into tissues or the cytoplasm. Insects belonging to Hemiptera, such as planthoppers, aphids, leafhoppers and whiteflies, have distinct piercing-sucking mouthparts 
that include needle-like stylet bundles and it is not surprising that Hemipteran insects transmit the majority $(>55 \%)$ of the vectored plant viruses [1]. The mechanisms of virus transmission are diverse. Based on differences in the length of the time that insects can harbor infectious viral particles, the transmission mechanisms can be classified into nonpersistent (harboring viruses for a few seconds/minutes), semipersistent (a few hours/days, but lost upon molting), and persistent (often throughout the lifespan of the vectors) [3]. Taking advantage of insect transmission, viruses can spread among plant individuals, usually resulting in epidemic outbreaks of viral disease and severe agricultural yield loss. Therefore, understanding the biology of vector insects and the viral transmission mechanisms will provide insight into interactions among insects, viruses, and plants, which will promote the development of effective techniques to prevent viral diseases of plants.

The small brown planthopper (Laodelphax striatellus Fallén), which was previously deposited in the deprecated, paraphyletic order Homoptera, is a notorious rice pest that is now classified into the Delphacidae of Hemiptera [4]. Besides injuring rice plants (Oryza sativa) by sap-sucking with its piercing-sucking mouthparts, $L$. striatellus also acts as the most important vector of rice stripe virus (RSV, belonging to Tenuivirus) in a persistent and propagative manner [5]. After invading L. striatellus, RSV can escape from the midgut, salivary, and ovary barriers and propagate in $L$. striatellus. Evidences have revealed that amorphous or filamentous inclusions of RSV exist in the cytoplasm of midgut epithelial cells, salivary glands, and the fat body. Moreover, it has been confirmed that RSV particles exist in follicular cells of the ovarioles and can be transmitted from female adults to their progeny via eggs [6]. Concomitant with the changes in global climate and agricultural systems, the damage caused by L. striatellus and RSV increased dramatically after 1960s. For example, in Jiangsu Province, one of the major grain producing areas in China, rice fields suffering from L. striatellus and RSV reached 1.57 million hectares in recent years, accounting for about $80 \%$ of the rice fields and causing $30-40 \%$ yield loss in that area [7]. In practice, controlling the outbreak of populations of $L$. striatellus is the most effective way to prevent RSV infection. As a consequence, L. striatellus has long been used as a model organism in the study of insect ecology, physiology, biochemistry, as well as molecular interactions between vectors and plant viruses.

Although L. striatellus is important in both theoretical and applied studies, genomic information for this insect is quite limited. For example, to date, there is no wholegenome sequencing project for this species, and only a few DNA sequences are available in the public databases $(<100$ entries). To collect genomic data efficiently, identi- fication of expressed sequence tags (ESTs) that represent the transcribed genes from a given set of tissues or individuals is a cost-effective approach. We employed massively parallel pyrosequencing on the Roche 454-GS-FLX platform to create EST databases of $L$. striatellus. In total we identified 41,492 different unigenes from both viruliferous and naïve L. striatellus. Gene Ontology (GO) classification indicated they were involved in various biological processes. Within these transcripts, we identified 1,451 potential microsatellite loci that can be used as DNA markers. Transcripts from symbiotic microorganisms, including RSV, Wolbachia and yeast-like symbiotes, were identified, and functional annotation revealed the possible lifestyle of these microorganisms within the insect cells. Comparison of our EST database with known insect immune systems showed that $L$. striatellus encodes similar immune regulatory systems, such as RNA interference, and JAK/STAT and Imd pathways that might be involved in resistance to viral infection [8-10]. In addition, comparative analysis of the two transcriptomes generated from viruliferous (RSV-infected) and naïve (noninfected) L. striatellus provided a list of candidate transcripts that potentially represent the biological response to viral infection. To our knowledge, this work is the first to study the transcriptome of L. striatellus. The transcriptional information provided will have an immediate effect on gene cloning, annotation, and functional studies of insect-plant-virus symbiosis.

\section{Results and Discussion}

\section{Pyrosequencing and contig assembly of ESTs}

As described in the Methods, viruliferous and naïve $L$. striatellus whole body cDNA libraries were subjected to a full-plate production run on the 454-GS-FLX sequencing instrument, resulting in 207,918 and 225,158 reads, respectively. The length distribution of these reads is depicted in Additional file 1. After filtering for adaptors and low-quality sequences, the two libraries generated 201,281 (viruliferous sample) and 218,681 reads (naïve sample), respectively, with a total of $96,567,749$ bases (Table 1). Files containing these data were deposited in the Short Read Archive of the National Center for Biotechnology Information (NCBI) with accession numbers of SRX016333 (viruliferous) and SRX016334 (naïve), respectively. As shown in Table 1, the general features of the contig assembly from the two libraries are similar: a total of 9,936 and 9,417 contigs were obtained from the viruliferous and naïve datasets, with average lengths of $376 \mathrm{bps}$ and $362 \mathrm{bps}$, respectively. When sequence data from the two libraries were combined, the average length of the high quality reads was $229 \mathrm{bp}$. These combined reads were assembled into 16,885 contigs with an average length of $384 \mathrm{bp}$ (ranging from 61 to 8,651 bp). In addition, there were 24,607 reads that failed to be covered by 
Table 1: General features of $L$. striatellus EST libraries

\begin{tabular}{lccc}
\hline & Viruliferous sample & Nä̈ve sample & Combined sample \\
\hline Total bases (bp) & 44741850 & 51825899 & 96567749 \\
High-quality reads ${ }^{\text {a }}$ & 201281 & 218681 & 419962 \\
Average reads length (bp) & 222 & 237 & 230 \\
Number of contigs & 9936 & 9417 & 16885 \\
$\quad$ Average contig length (bp) & 376 & 362 & 384 \\
Range of contig length (bp) & $60 \sim 3752$ & $61 \sim 4182$ & $61 \sim 8651$ \\
Number of reads in contigs & 174662 & 165212 & 383067 \\
Number of singletons & 14331 & 14117 & 24607 \\
Unigenes (contigs + singletons) & 24267 & 23534 & 41492 \\
Matched CDSs b & 22664 & 22447 & 38019 \\
Specific sequences & 15746 & 14318 & - \\
\hline
\end{tabular}

a. Adaptors and low-quality reads were excluded.

b. Matched CDS means the number of predicted protein coding sequences from unigenes.

contigs and were treated as singletons. In total, 41,492 unigenes were obtained from the combined EST library. All files of assembled contigs and singletons from viruliferous, naïve, and combined EST libraries are available by request.

\section{Similarity searches and gene ontology analysis}

ESTs were subjected to BlastX similarity search against the NCBI-NR protein database to determine their putative function. As shown in Fig. 1A, among the 41,492 unique sequences that contain ESTs from both viruliferous and naïve $L$. striatellus, 6,873 (16.6\%) showed significant matches at the cutoff e-value $\leq 10^{-3}$, whereas 5,659 (13.6\%) showed poor matches with e-values between $10^{-3}$ and 10. The remaining 28,960 (69.8\%) ESTs exhibited no useful matches (e-value $>10)$. As a result, we estimated that the majority of the genes of $L$. striatellus ( $>80 \%$ ) are function-unknown. To compare the similarity of L. striatellus genes with those of the other arthropods, EST sequences were also subjected to BlastX search against putative protein sequences (e-value $\leq 10^{-3}$ ) of eleven species of arthropod whose complete or draft genomes are available. As shown in Fig. 1B, the smallest number of database matches $(8,959 ; 21.6 \%)$ was from deer tick (Ixodes scapularis). This can be explained by the fact that I. scapularis belongs to Arachnida and is not a species of Insecta. The other species belong to Insecta are phylogenetically distributed into Phthiraptera (Pediculus humanus), Coleoptera (Tribolium castaneum), Lepidoptera (Bombyx mori), Hymenoptera (Apis mellifera and Nasonia vitripennis), Diptera (Drosophila melanogaster, Anopheles gambiae, Culex pipiens and Aedes aegypti), and Hemiptera (Acyrthosiphon pisum); however, the numbers of matched sequences are very similar (from
10,290 - 11,646). Unexpectedly, although pea aphid (A. pisum) belongs to Hemiptera, as does L. striatellus, and currently its draft genome has the largest set of putative protein coding sequences $(37,994)$, only $10,979(26.5 \%)$ ESTs of $L$. striatellus were found to be similar to the proteome of $A$. pisum. This number is smaller than that of parasitoid of flies ( $N$. vitripennis, 11,162; 26.9\%), head louse (P. humanus, 11,203; 27.0\%), honey bee (A. mellifera, 11,321; 27.3\%), and red flour beetle (T. castaneum, $11,646 ; 28.1 \%)$. The number of matched sequences does not entirely reflect the phylogenetic relationships and genome sizes. This discordance might be simply attributed to the reason that our understanding of the genomes of insect is relatively inadequate.

To assess whether our EST libraries were substantial representative samples of the transcriptome of $L$. striatellus, the search results of BlastX against the Swiss-Prot/ TrEMBL database were combined, and matches were assigned GO (gene ontology) categories with biological process, molecular function, and cellular components [11], respectively. Fig. 2 depicts the results of GO classification of molecular function and reveals that, for EST libraries of viruliferous, naïve, and combined samples, the percentages of transcripts in each GO category are quite similar. This is also true for those ESTs classified as biological process as well as cellular components. These results suggested that our EST libraries sampled widely across the GO sub-categories and provided a good representation of the L. striatellus transcriptome.

The coverage of the combined EST library of L. striatellus was assessed by two analyses. Firstly, 366 contigs and singletons of this library are found to encode possible ribosomal proteins. We then compared these sequences by BlastX search against a database of the ribosomal pro- 


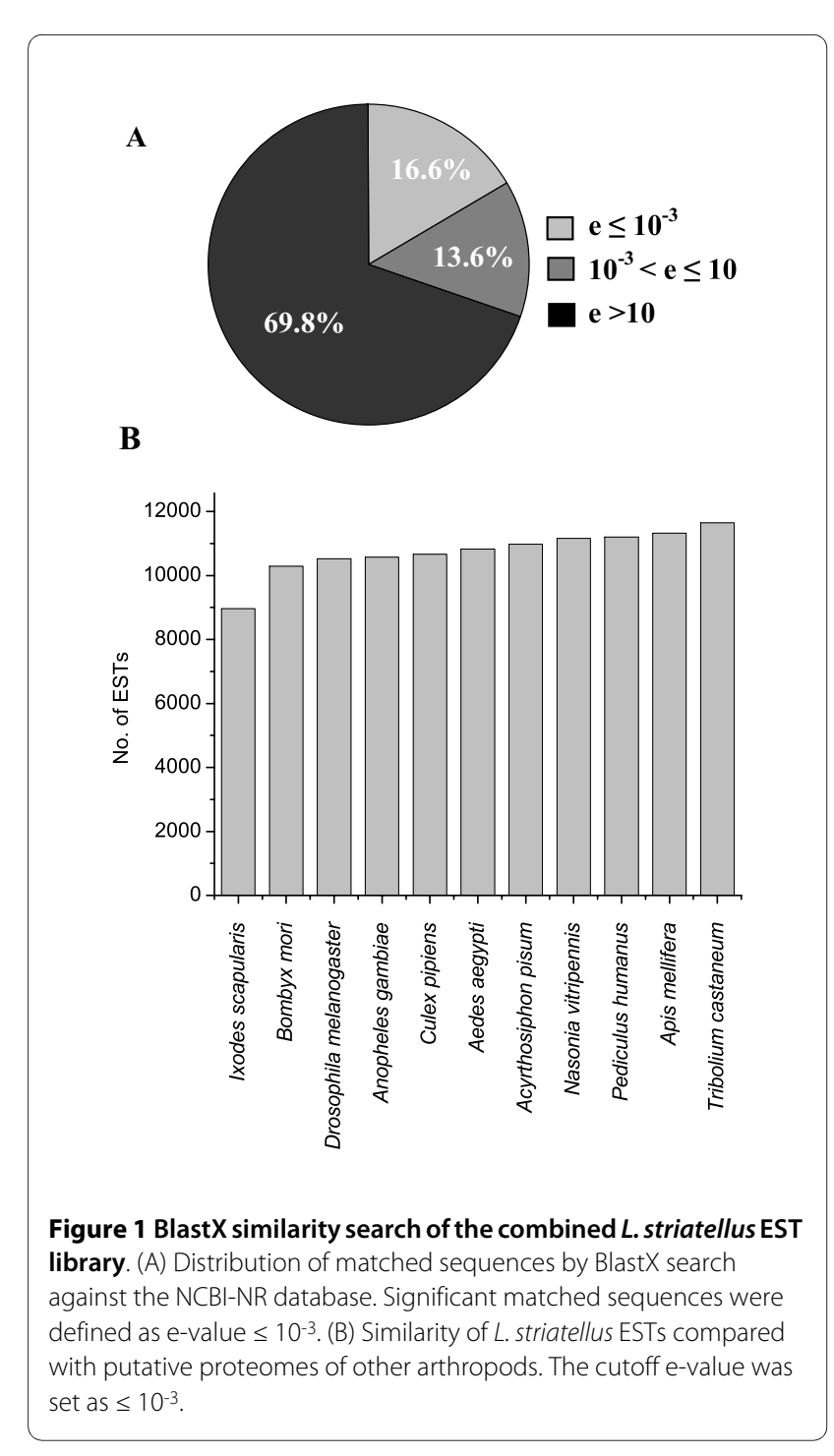

teins of $D$. melanogaster (e-value $<10^{-3}$ ), which contains 88 cytoplasmic ribosomal proteins and 79 mitochondrial ribosomal proteins. Except four ribosomal proteins of $D$. melanogaster (ribosomal protein L41, mitochondrial ribosomal protein S5, S31 and L51), the others have significantly matched orthologs in the EST library of $L$. striatellus. The second analysis used the 41,492 unigenes of L. striatellus to search against recently reported protein sequences of its close-relative pea aphid (OGS 1.0 of $A$. pisium, including 34,821 sequences from NCBI RefSeq plus non redundant GLEAN) [12]. A total of 12,361 nonredundant protein sequences of $A$. pisium exhibited significant similarity to the sequences from L. striatellus (BlastX, e-value $<10^{-3}$ ). The remaining unigenes that did not show significant matches to $A$. pisum, were used to search again against the protein sequences of $D$. melanogaster (containing 21,603 sequences). An extra 1,719 hits were obtained, suggesting that the combined transcrip- tome of L. striatellus has at least 14,080 protein coding genes (12,361 from $A$. pisium plus 1,719 from $D$. melanogaster). We suppose that the remaining $\sim 20,000$ unigenes which did not return significant BlastX hit may encode previously uncharacterized proteins, non-coding RNAs, or products from microbial endosymbionts.

\section{Identification of microsatellites}

Microsatellites (or simple sequence repeats, SSRs) are hyper-polymorphic DNA fragments that contain repeating units of 1 - 6 base pairs [13]. Although they are widely used as molecular markers, no microsatellite sequences have been reported for L. striatellus. We identified many microsatellite loci with di-, tri-, tetra-, penta- and hexanucleotide repeats (minimum repeats $>6$ ) from the combined EST library using SciRoKo ver 3.4 software [14]. Table 2 shows that, when perfect repeat motifs were considered, a total of 423 microsatellite markers were identified (ranging from 15 - $128 \mathrm{bps}$ ) and the mean microsatellite density is one per $27.7 \mathrm{~kb}$. Among these molecular markers, tri-nucleotide repeats are predominant $(76.6 \%)$, with (AAC)n being the most frequent motif $(32.4 \%)$. When a conserved degree of base-pair mismatch ( $\leq 2 \mathrm{bps}$ ) was applied in the repeat motif search, a total of 1,451 microsatellite loci were identified (ranging from 15 - 132 bps) and the mean microsatellite density was one per $8.07 \mathrm{~kb}$. Of them, only 564 (38.9\%) were found in protein coding transcripts, including those annotated as hypothetical and conserved hypothetical proteins. Similar to the perfect repeats, tri-nucleotide repeats are also predominant (56.2\%) and the motif (AAC)n (18.7\%) is the most frequent, followed by (AAG)n (8.2\%), (AAT)n (7.7\%), (AGC)n (6.3\%) and (AGG)n (5.1\%). Among all possible di-nucleotide microsatellites $[(\mathrm{CA}) \mathrm{n},(\mathrm{GA}) \mathrm{n}$, (AT)n and (GC)n], no perfect or imperfect (GC)n sequence repeat was found in EST library of L. striatellus. These identified microsatellites have the potential to be used in genetic mapping, parentage analysis, genotyping, gene flow, and in population genetics.

\section{Identification of transcripts of endosymbiotic microbes Rice stripe virus (RSV)}

RSV is the type member of the genus Tenuivirus that has an unusual thread-like morphology under the electron microscope [5]. The RSV genome contains four segmented single-stranded RNAs (ssRNAs), which encode seven open reading frames (ORFs). Among them, RNA1 encodes a part of the RNA-dependent RNA polymerase (RdRP)[15]. RNA2, RNA3, and RNA4 are ambisense. Each of them has two ORFs: one located on the 5' part of the viral RNA and the other on the $5^{\prime}$ part of the viral cRNA. RNA2 encodes a function-unknown protein (NS2) and a putative membrane glycoprotein, NSvc2. RNA3 encodes a suppressor protein (NS3) and a nucleo- 


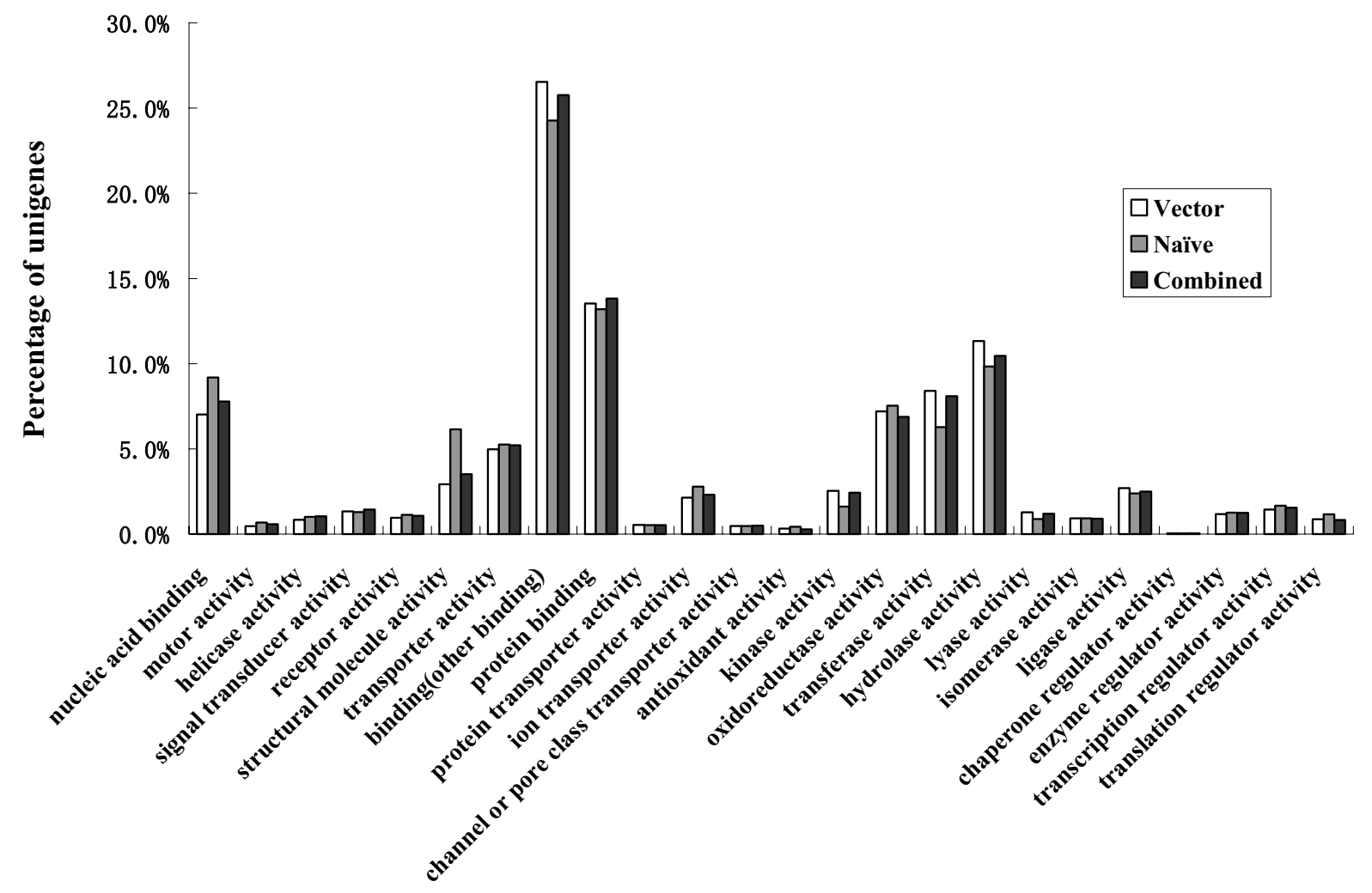

Figure 2 Comparison of the distribution of GO terms. The X-axis shows subgroups of molecular functions from GO, the Y-axis shows the percentage of the matched EST sequences. Distribution of GO terms of ESTs from viruliferous (vector), naïve, and combined samples are compared.

capsid, NCP. RNA4 encodes a nonstructural disease-specific protein (SP) and a movement protein, NSvc4 $[5,16]$. To investigate the expression level of these ORFs in $L$. striatellus, each RSV ORF sequence was searched against our EST libraries. No RSV transcript was found in the ESTs of naïve insects, confirming the naivety of this sample. For ESTs of the viruliferous insect, the abundance of RSV transcripts is shown in Fig. 3. NS3 is the most abundant transcript (46 reads in total) and is a $23 \mathrm{kDa}$ protein that was experimentally demonstrated as a suppressor of gene silencing because it can significantly reduce the accumulation of small interference RNA (siRNA) in plant cells, and can bind 21-nucleotide single-strand (ss) siRNA, siRNA duplexes, and long ssRNA [17]. The dominant presence of the NS3 transcript suggests that after invading L. striatellus, RSV must suppress the immune response of the host insect as well as the host plant. Therefore, it would be interesting to identify the host cellular target that is suppressed by RSV NS3 in a future study. The other transcripts, including RdRP, NS2, NSvc2, NCP, and SP, were present in very low levels in the EST library of L. striatellus (matched reads from 1-5), suggesting that replication and assembly of RSV is not very active in the insect. In addition, we failed to identify transcripts of the movement protein NSvc4, which agrees with the existing hypothesis that the movement protein usually functions in planta during virus spreading to neighboring cells through the plasmodesmata [16], and thus its expression might be shut down or at a very low level in the insect.

\section{Wolbachia}

Wolbachia are obligatory, intracellular, Gram-negative bacteria that infect a wide range of arthropods and nematodes [18]. Wolbachia can enhance their maternal transmission by manipulating host reproductive systems through strategies such as cytoplasmic incompatibility, parthenogenesis, feminization, and male killing [7]. Interestingly, it has been recently discovered that this bacterial endosymbiont can reduce the RNA virus load in D. melanogaster and thus promote the antiviral potential of host insect $[19,20]$. In L. striatellus, Wolbachia infection is familiar and it was reported that the bacterium is distributed in the head, thorax, abdomen, salivary gland, guts, ovary, and testis [21]. To investigate whether our samples 
Table 2: Statistics of microsatellite loci derived from the EST library

\begin{tabular}{ccccccccc}
\hline \multicolumn{4}{c}{} & \multicolumn{3}{c}{ Perfect repeats } & \multicolumn{3}{c}{ Imperfect repeats a } \\
\hline $\begin{array}{c}\text { Repeat } \\
\text { size }\end{array}$ & No. & $\begin{array}{c}\text { Average } \\
\text { length (bp) }\end{array}$ & $\begin{array}{c}\text { GC } \\
\text { content }\end{array}$ & No./Mb & No. & $\begin{array}{c}\text { Average } \\
\text { length (bp) }\end{array}$ & GC content & No./Mb \\
\hline 2 & 76 & $18.7 \pm 5.07$ & 0.43 & 6.49 & 88 & $21.9 \pm 7.74$ & 0.43 & 7.51 \\
3 & 324 & $24.0 \pm 10.31$ & 0.39 & 27.66 & 816 & $22.1 \pm 10.48$ & 0.40 & 69.66 \\
4 & 14 & $32.1 \pm 11.51$ & 0.20 & 1.20 & 169 & $20.2 \pm 9.51$ & 0.17 & 14.43 \\
5 & 3 & $32.0 \pm 2.83$ & 0.06 & 0.26 & 242 & $17.7 \pm 4.01$ & 0.32 & 20.66 \\
6 & 6 & $57.2 \pm 23.07$ & 0.37 & 0.51 & 136 & $30.4 \pm 21.62$ & 0.48 & 11.61 \\
\hline
\end{tabular}

a. The conservative degree of mismatch of repeats was set as $\leq 2 \mathrm{bp}$.

b. No./Mb represents the average number of microsatellites per $1 \mathrm{Mb}$ of EST sequence the library.

are infected by Wolbachia and to determine the gene expression pattern of the bacterium, we searched the EST libraries and annotated a total of 103 Wolbachia genes. Both viruliferous and naïve EST libraries contain transcripts of Wolbachia, and the number of matched reads in the naïve sample is four times larger than that of the viruliferous sample (528 vs. 132 reads). It is not clear whether this expression bias is associated with RSV infection.

The available sequenced genomes of Wolbachia encode approximately 1,000-1,400 proteins [22]; therefore, we estimated that the Wolbachia transcripts identified in our study represent about $10-13 \%$ of the transcriptome of the bacteria. Additional file 2 shows that the annotated Wol-

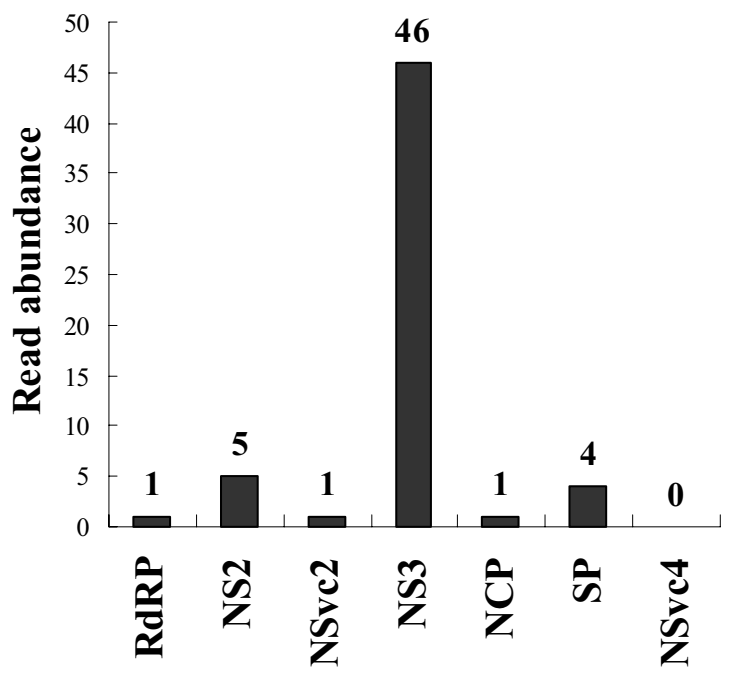

Figure 3 Abundances of transcripts of RSV ORFs. RdRP: RNA dependent RNA polymerase; NS2: function-unknown protein; NSvc2: putative glycoprotein; NS3: RNAi suppressor; NCP: nucleocapsid; SP: disease-specific protein; NSvc4: movement protein. The Y-axis shows the number of reads identified in the viruliferous EST library. bachia genes can be classified into four major functional categories. (1) Function-unknown genes: this group contains 53 transcripts $(51.5 \%)$ of Wolbachia, including the first three most abundant genes. (2) Genes essential for cell processes such as DNA and RNA synthesis, ribosomal assembly, and electronic transportation. (3) Genes responsible for de novo biosynthesis of nucleotides and cofactors, including purine, riboflavin, coenzyme A, and ubiquinone. Identification of these gene transcripts suggests that Wolbachia cannot obtain these chemicals from the host insect cells and have to synthesize them themselves, as predicted by genomic annotation [7]. (4) Gene products involved in transport, including an $\mathrm{ABC}$ transporter, proprotein translocase, permease, $\mathrm{Na}^{+} / \mathrm{H}^{+}$antiporter, and a set of chaperones (DnaK, GroES, and a cold shock protein). Among them, a gene encoding multidrug resistance protein $\mathrm{D}$ was identified. The product of this gene has an AcrF-like domain and belongs to the RND efflux pump family [23]. We hypothesize that the protein is responsible for pumping harmful host-derived chemicals out of the bacterial cell envelope to maintain homeostasis. Besides the above-mentioned genes, an ATPdependent protease, Lon, was identified. In bacteria, this kind of protease degrades naturally unstable proteins and thus takes part in diverse cell processes. It has been reported that inactivation of Lon genes will attenuate the virulence of various pathogenic bacteria, such as Salmonella enterica, Yersinia pestis, and Pseudomonas syringae [24]. Based on this, we speculate that Lon of Wolbachia plays an important role in symbiosis with $L$. striatellus. Taken together, these identified Wolbachia genes may reveal facets of how an endosymbiotic bacterium adapts to the living environment within cells of host insects.

\section{Yeast-like symbiotes (YLS)}

The YLS are microbial, transovarially inherited endosymbiotes being found in the fat body of Laodelphax striatellus. Phylogenetic analysis has revealed that the YLS consists of fungal species from the ascomycetes and 
pyrenomycetes $[25,26]$. These endosymbiotes can benefit their hosts by providing nutrition such as vitamins and sterols, and loss of the YLS under high temperature can cause deleterious effect on the host insect [27]. In this study, although the whole bodies, rather than the fat bodies of insects, were used as samples to generate EST sequences, and the strategy constructing cDNA libraries (see Methods) has pre-excluded the large-scale contamination of $18 \mathrm{~S}$ rRNA sequences that can be used to identify the species of YLS, close examination of the combined EST library retrieved 3,061 unigenes that matched well with the fungal species belonging to 45 genera (Additional file 3). Among them, 2,028 unigenes exhibit significantly matches (Blastx search, e-value $<10$ $3)$, and the majority of these unigenes $(2,637,86.1 \%)$ encode function-unknown proteins. For lacking of $18 \mathrm{~S}$ rRNA sequences, it is hard to determine whether a unigene derives from the YLS. However, among the matched 45 fungal genera, 20 of them have been previously reported to be the YLS and their transcripts can provide useful information [25,26]. For example, in our library there are 44 unigenes matched well with Aspergillus fumigatus. Among them, seven genes encode products that may be involved in cell signaling, including two zinc finger proteins, two ankyrin-repeat domain containing proteins, a RING-finger protein and two proteins related to the ubiquitin-mediated protein degradation. Future functional studies will reveal whether they have association with the regulation of expression of the symbiosisrelated genes.

\section{Putative immune regulatory proteins}

One of the long-term goals in studying vector-virus symbiosis is to elucidate the molecular mechanisms by which viruses manipulate the host innate immune system to avoid injury from the defensive response. Therefore, understanding the host immune system is fundamental to answering this question. By comparing our EST database with known immune proteins encoded by other insects, especially $D$. melanogaster, putative regulatory pathways of L. striatellus immune system were annotated. Among them, we focused on antivirus related cascades, including RNAi silencing, JAK/STAT, and Imd pathways (Table 3).

\section{Putative $R N A$ silencing related proteins}

Currently RNA interference (RNAi) is the only mechanism that has been revealed to target viruses directly in insects $[9,28]$. The model insect $D$. melanogaster encodes two Dicers (Dicer-1 and Dicer-2), five Argonautes (AGO$1 \sim$ AGO3, PIWI, and Aubergine), and two dsRNA-binding proteins (dsRBP, including R2D2 and R3D1) that are involved in RNAi. Among them, Dicer-2, AGO-2, and R2D2 are responsible for the antiviral response. After viral double-stranded RNAs (dsRNAs) are recognized, RNAse III enzyme Dicer-2 forms a complex with dsRBP
R2D2, and then cleaves newly synthesized viral dsRNA to produce small interfering RNAs (siRNAs) that guide the AGO-2 containing RISC complex to specifically recognize and degrade viral RNA [29]. Among the ESTs of $L$. striatellus, five and seven contigs that might encode Dicer- and AGO-like proteins, respectively, were identified (Table 3). However, because Dicer and AGO proteins are similar in structure and our transcripts are not fulllength, it is difficult to discriminate these Dicer- and AGO-like proteins into different sub-classes. In addition, we obtained an R3D1 homologous transcript (FQ92HJ001EMI2C) but failed to retrieve any R2D2 homologs from our database. Comparative analysis of EST abundance showed that there was no substantial difference in the levels of putative RNAi related genes between viruliferous and naïve samples of L. striatellus. This is in accordance with previous results showing that, even when challenged with pathogenic viruses, insect RNAi pathway genes are constitutively expressed and are not upregulated by viral infection [30,31]. Consequently, which RNAi process of L. striatellus is suppressed by RSV is an open question.

\section{Putative JAK/STAT proteins}

The JAK/STAT pathway consists of four major components: the ligand UPD, the receptor Domeless, the Janus kinase (JAK), and the signal transduction and activators of transcription (STAT) $[9,32]$. After receiving a specific signal, UPD binds to the fat body receptor Domeless and activates phosphorylation of the non-receptor tyrosine kinase JAK. In due course, STAT is phosphorylated by JAK to form dimmers and is recruited into the nucleus where it binds to palindromic region of promoters of downstream genes to activate their transcription [33]. In D. melanogaster, the JAK/STAT pathway is usually involved in differentiation of hemocytes and resistance to bacterial or fungal infection. However, evidences have accumulated that reveal that this pathway is also associated with the antiviral response, possibly through sensing cytokine signals and inducing antiviral response genes $[30,34]$. Using the above-mentioned proteins of D. melanogaster as search queries, we found that L. striatellus encodes homologs of JAK, Domeless, and STAT (Table 3 ), suggesting it contains a JAK/STAT cascade and that these matched proteins are conserved during insect evolution. However, no UPD transcript (UPD, UPD2, or UPD3) was found. This can be explained by that UPD transcripts are not present in our EST library or L. striatellus does not encode similar proteins. Considering that many other insects whose genomes have been sequenced (such as $A$. gambiae and B. mori) also lack UPD-like genes, we believe that the JAK/STAT pathway of L. striatellus might be activated by sensing ligands that differ from UPD of D. melanogaster. 
Table 3: Putative immune regulatory genes of $L$. striatellus that might be involved in resistance to viral infection.

\begin{tabular}{|c|c|c|}
\hline Regulatory protein & Function and description & Code of ESTs (e-value) \\
\hline \multicolumn{3}{|c|}{ RNAi systems } \\
\hline \multirow[t]{4}{*}{ Dicer like protein } & $\begin{array}{l}\text { RNAIll family endo-ribonucleases that cleave double-stranded } \\
\text { RNA and pre-microRNA into short dsRNA fragments. }\end{array}$ & Contig2793 (2e-27), Contig6128 (6e-06), \\
\hline & & FQ4QJ5301BI24S (6e-17), \\
\hline & & FQ92HJ001CXB6B (2e-13), \\
\hline & & FQ4QJ5301EGA00 (7e-09) \\
\hline \multirow[t]{6}{*}{ Argonaute like protein } & Catalytic component of the RNA-induced silencing complex & Contig1 164 (7e-24), Contig6509 (2e-18), \\
\hline & & Contig15043 (2e-21), \\
\hline & & FQ4QJ5301BLRSO (5e-38), \\
\hline & & FQ92HJ001AVX3O (4e-16), \\
\hline & & Q92HJ001B74KA (1e-10), \\
\hline & & FQ92HJ001DDNCB (1e-11) \\
\hline $\mathrm{R} 2 \mathrm{D} 2$ & dsRNA-binding proteins & No match ${ }^{b}$ \\
\hline R3D1 & dsRNA-binding proteins & FQ92HJ001EMI2C (1e-05) \\
\hline \multicolumn{3}{|c|}{ JAK/STAT pathway } \\
\hline UPD-like protein & Ligand activating JAK/STAT pathway & No match ${ }^{b}$ \\
\hline Domeless & Transmembrane cytokine receptor & FQ92HJ001EGTO6 (0.99) \\
\hline Hopscotch & Janus Kinases (JAK) that have tyrosine kinase activity & Not determined $c$ \\
\hline STAT & $\begin{array}{l}\text { SH2-domain containing protein that can be phosphorylated } \\
\text { by JAK }\end{array}$ & FQ4QJ5301ERDD2 (1e-15) \\
\hline \multicolumn{3}{|c|}{ Imd pathway } \\
\hline Imd & $\begin{array}{l}\text { Death domain-containing protein that similar to receptor } \\
\text { interacting protein (RIP) of TNF-R pathway }\end{array}$ & FQ92HJ001A68SH (0.031) \\
\hline \multirow[t]{2}{*}{ PGRP } & Peptidoglycan recognition protein & Contig145 (6e-23), Contig6140 (1e-26), \\
\hline & & Contig6821 (3e-37) \\
\hline Relish a & NF-kB-like transcription factor & FQ4QJ5301E34NM (0.014) \\
\hline MAP3K, TAK1 & Mitogen-activated protein 3 kinase & Not determined $\mathrm{c}$ \\
\hline TAB2 & TAK1 binding protein & FQ4QJ5301DF7ET (1e-18) \\
\hline DIAP2 & Inhibitor of apoptosis proteins & Contig3110 (1e-21) \\
\hline IKK $\beta /$ ird5 & Inhibitor of NF-KB kinase & Not determined $c$ \\
\hline IKKY/Kenny & Inhibitor of NF-KB kinase & Not determined $c$ \\
\hline dFADD & Fas-associated death domain protein & FQ92HJ001A68SH (0.031) \\
\hline Dredd caspase & A caspase involved in apoptosis & No match ${ }^{b}$ \\
\hline
\end{tabular}

a. The protein Relish is usually regarded as a component of the NF-KB pathway.

b. No significant match $\left(e \leq 10^{-3}\right)$ in searching EST libraries of $L$. striatellus.

c. This indicates that although there are significant matches, but it is hard to determine the preferred one since these matched proteins are highly similar in predicted secondary structure.

\section{Putative Imd pathway proteins}

The Imd pathway regulates the expression of antimicrobial peptide (AMP) genes by perception of signals from pathogen-associated molecular patterns (PAMPs), such as the diaminopimelic acid (DAP)-type peptidoglycan of Gram-negative bacteria [35]. The Imd pathway can be activated through peptidoglycan recognition proteins PGRP-LC and PGRP-LE [36]. After receiving signals from PAMPs, these receptors drive the activation of a critical NF- $\kappa B-l i k e$ transcription factor Relish (Rel) through a complicated signaling cascade that consists of IMD, dTAK1, dTAB2, IKK $\gamma$, IKK $\beta$, ird5, and dFADD proteins to modulate AMP gene expression [37]. Studies revealed that mutations in several Imd pathway genes, including PGRP-LC, Tak1, ird5, key, and rel genes (but not imd and $d F A D D$ ), resulted in high levels of suscepti- 
bility to viral infection in D. melanogaster [38,39]. Moreover, the mammalian tumor necrosis factor receptor (TNFR) pathway, which is the counterpart of the insect Imd pathway, has long been known to regulate the immune response to viral infections [40]. These results indicated that a branch of the Imd pathway is also required in defense against viruses. Interestingly, recent genomic annotation of pea aphid ( $A$. pisum), a close relative of L. striatellus in phylogeny, revealed that this insect lacks the principal Imd, dFADD, Dredd, Relish and even PGRP proteins [41]. In the EST libraries of L. striatellus, homologs of Imd pathway gene transcripts, including Imd, TAB2, DIAP2, dFADD, and NF- $\mathrm{kB}$ like protein Relish, were identified, but it is noticeable that the homologs of Imd, Relish and dFADD have a relatively lower matched score (Table 3). In addition, the PGRP-like proteins are present in the EST libraries, which is different from that of the A. pisum (Table 3). Furthermore, although there are a number of matched targets that are similar to protein kinases TAK1, IKK $\gamma$, and $\mathrm{IKK} \beta$, it is hard to separate these transcripts from other kind of kinases because their predicted secondary structures (by pfam search, e $<0.1$ ) are quite similar. In addition, we failed to identify a homolog of the Dredd caspase (Table 3 ). Taken the above information together, we suppose that the Imd pathway of L. striatellus, if it exists, is different from that of $D$. melanogaster and $A$. pisum. It will be interesting to determine whether this genetically differentiated immune pathway also has an association in defending RSV infection.

\section{Comparative analyses of EST libraries}

There were 15,746 and 14,318 unigenes specifically identified in viruliferous and naïve EST databases, respectively (Table 1). However, due to the non-saturated nature of our EST databases and the relatively short average unigene length derived from pyrosequencing, these specific sequences cannot be a reliable representation of the transcriptional differences between the different samples. Therefore, we compared the differences in transcript abundance between viruliferous and naïve $L$. striatellus. Additional file 4 lists the top 30 contigs that are more abundant in the viruliferous sample. Although care must be taken in draw the conclusion that highly abundant ESTs play critical roles in symbiosis with RSV, the analysis provided a candidate gene list to further investigate the molecular basis of L. striatellus - RSV interactions.

It is interesting to note that transcripts encoding vitellogenin are the most abundant in the viruliferous insect sample (Additional file 4). We then retrieved all 34 unigenes of L. striatellus encoding vitellogenin and mapped them onto their ortholog (BAF75351.1) of brown planthopper (Nilaparvata lugens) by BlastX. The protein products of these unigenes have overlapped regions and a plenty of single nucleotide polymorphism were identified within these regions, indicating that L. striatellus contains multiple copies of vitellogenin genes. Vitellogenin is an egg yolk precursor for lipoproteins and phosphoproteins that is expressed in female insects. The protein is synthesized extraovarially in the fat body, transported through the hemolymph, and eventually internalized by competent oocytes through membrane-bound receptors named vitellogenin/lipophorin receptors [42]. Thus, it was suggested that the vitellogenin traffic pathway might be exploited by viruses to overcome the physical barrier between follicle cells and oocytes [43]. In fact, in D. melanogaster, it has been observed that when genes responsible for endosomal trafficking of vitellogenin from the follicle cells to the oocyte were mutated, the transport of retrovirus ZAM viral particles from follicle cells to oocytes was arrested. This result strongly supported the hypothesis that ZAM uses the vitellogenin traffic pathway to gain access to the oocyte to affect the maternal germ line [44]. Similarly, the extraordinary abundance of vitellogenin transcripts in viruliferous $L$. striatellus EST library suggested that RSV might modulate the host vitellogenin pathway genes to facilitate its persistent transmission via the insect ovum.

\section{Expression profiling by RT-PCR}

Semi-quantitative RT-PCR analysis was used to confirm the expression of 52 selected contigs of L. striatellus. Most of these unigenes are selected because they exhibited high abundance in the EST library of viruliferous samples. A non-structural protein gene (Contig12404) that was supposed to be encoded by Himetobi P virus, another virus being reported to infect $L$. striatellus, was also included. The results of amplification showed that 45 of the transcripts (Additional file 5) are indeed expressed in both viruliferous and naive L. striatellus (Fig. 4), demonstrating that they are not sequencing artifacts. This strongly supports the view that the parallel sequencing technique is a quick and highly effective approach for investigating the transcriptome of poorly-understand organisms.

Among successfully amplified contigs, 18 candidate genes showed reproducibly up-regulated expression patterns in viruliferous insects (Fig. 4), including genes encoding transferrin (Contig3086 and Contig6985), ribosomal protein L4 (Contig2551), ribosomal protein L15 (Contig5503), G-protein (Contig1243), malic enzyme (Contig7591), ATP-citrate synthase (Contig2280), and mitochondrial ATP synthase alpha subunit (Contig2413). The expression patterns are in accordance with the reads abundance of pyrosequencing, suggesting that these genes are indeed upregulated in viruliferous L. striatellus. Before in-depth functional study being carried out, how did these genes relate to symbiosis with RSV remains illu- 
sive. For example, it has been found that ribosomal protein S3 (RPS3) is a subunit of NF- $\mathrm{kB}$ transcriptional complexes in vertebrate. It forms a complex with NF-kB subunit p 65 and promotes the selective affinity of NF-kB complexes for activating the expression of a group of immune-related genes [45]. This implies that ribosomal protein may be involved in regulating immune response by protein-DNA interactions. Our RT-PCR assay confirmed that two ribosomal large-subunit proteins, L4 and L15, were up-regulated in the viruliferous insects. The two proteins have quite different secondary structures compared with the RPS3 so that how they are involved in innate immunity system of $L$. striatellus will be an opening question.

Both Contig3086 and Contig6985 encode transferrins. Of them, there is no read of Contig6985 identified in the naïve insect samples whereas 300 reads were found in the viruliferous samples. Transferrins are glycoproteins that bind iron ion with high affinity. These proteins often take part in antibacterial response since they decrease the concentration of available iron ions in the animal tissues so that pathogenic bacteria can not grow and proliferate because the starvation of iron. However, excess cellular iron is also associated with increased infection rate for viral diseases such as acquired immune deficiency syndrome (AIDS) caused by human immunodeficiency virus (HIV) and hepatitis caused by hepatitis $\mathrm{C}$ virus (HCV) [46]. Viruses can affect the expression of host proteins involved in iron homeostasis and benefit from iron overload. For example, the HIV-1 Nef protein regulates the localization of HFE, a host nonclassical major histocompatibility complex $(\mathrm{MHC})$ class I protein that binds to transferrin receptors. This process causes accumulation of iron in macrophages [47]. In addition, if the concentration of cellular iron is low, the activity of iron-dependent viral enzymes, such as ribonucleotide reductase, will be decreased and then through negative impact on viral rep-

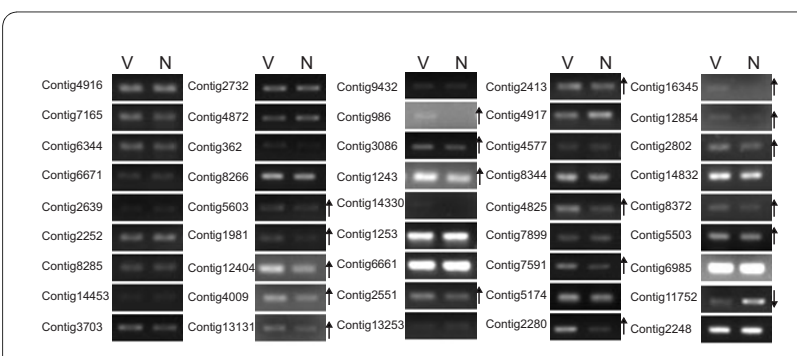

Figure 4 RT-PCR profiles of putative transcripts from viruliferous and naïve $L$. striatellus samples. V: viruliferous; N: naïve. The arrow next to the gel pictures indicates increased or decreased expression of a particular gene in viruliferous vs. naïve sample. At least two rounds of independent replication were used for each primer pair. The putative elongation factor gene (Contig2248) was used as a reference. The sequences of PCR primers used in this analysis are listed in Additional file 6. lication [48]. Based on this, it will be interesting to determine the functional role of these transferrin genes during RSV infection and how they are up-regulated.

\section{Conclusions}

Understanding the molecular interactions between $L$. striatellus and its symbiotic microbes is useful, not only for studies on the biology of species relationships, but also for agricultural practice that aims to develop effective strategies to prevent viral disease via controlling vector insects. However, these studies have been hampered by the lack of genomic resources of L. striatellus. Here, we employed the massively parallel pyrosequencing technique to collect ESTs from viruliferous and naïve samples of $L$. striatellus. When these ESTs were combined, 16,885 contigs and 24,607 singletons were generated, of which about $16.6 \%$ can be assigned a biological function. This genome-scale transcriptional information provides a basis for further investigation of the biology of L. striatellus. For example, we annotated a set of putative innate immunity regulatory genes that are similar to those of well-defined insects, including RNAi, JAK/STAT, and Imd pathways genes. This result will facilitate future studies on investigating the immune response of L. striatellus to microbial attack. Transcripts from RSV, the endosymbiotic bacteria Wolbachia and the yeast-like symbiotes were identified from our EST libraries. Functional annotation of these genes indicates that microbial processes, such as suppressor activity, de novo biosynthesis of cofactors, as well as molecule transportation, are critical for their survival in cells of L. striatellus. In addition, comparative genomic analysis advanced a repertoire of candidate genes that might be involved in the L. striatellus - RSV interaction. At present we are using more precise methods to investigate the functional roles of these candidate genes during RSV infection and transmission.

\section{Methods}

\section{Insect rearing and RNA isolation}

The culture of L. striatellus used in this study was collected from Jiangsu Province, China, and was maintained in laboratory for nearly six years. Both viruliferous and naïve $L$. striatellus were reared separately in glass beakers as stock populations. Rice (Oryza sativa cv. Nipponbare) plants were used as the planthoppers' diet throughout this experiment. Plants were grown in soil at $25^{\circ} \mathrm{C}$ under a long day photoperiod of $16 \mathrm{~h}$ under light and $8 \mathrm{~h}$ under dark in a growth incubator. The beakers were enclosed with a piece of nylon mesh after insects had been introduced into $2-3 \mathrm{~cm}$ high rice seedlings. The planthoppers were transferred to fresh seedlings every 10 - 14 days to assure sufficient nutrition. 
For each sample, approximately 300 insect individuals were used for RNA extraction. The insects were anesthetized with ether and frozen with liquid nitrogen in mortars. Total RNA was isolated from whole bodies collected from populations of viruliferous and naïve insect populations, respectively, following the standard protocol of TRIzol reagent (Invitrogen). The concentration and quality of total RNA were determined by a NanoDrop spectrophotometer (Thermo Scientific).

\section{cDNA library construction and sequencing}

Double-stranded cDNA was synthesized from the two RNA pools according to $\mathrm{Ng}$ ' s full-length cDNA synthesis protocol [49] with modifications. A GsuI-oligo dT primer was used to prime first-strand cDNA synthesis from 20 $\mu \mathrm{g}$ of mRNA, using 1,000 units of Superscript II reverse transcriptase (Invitrogen). The diol group of the CAP structure of mRNA was then oxidized by NaIO4, followed by biotinylation with biotin hydrazide (long arm, Vector Laboratories). After RNaseONE digestion, Dynal M280 streptavidin Dynabeads were used to select biotinylated RNA/cDNA. The first-strand cDNA was then released by alkaline hydrolysis, and two 5 ' adaptors (N5 and N6 adaptors) were ligated to the 5 '-end of the firststrand cDNA. Double-stranded cDNA was synthesized by primer extension using Ex Taq polymerase (TaKaRa). The polyA tail was removed by GsuI digestion, and cDNA size fractionation was performed using a cDNA size fractionation column (Invitrogen). Each cDNA fraction larger than $800 \mathrm{bp}$ was sonicated to the range of 300$800 \mathrm{bp}$, and then pooled together with the other cDNA samples ranging from $300 \mathrm{bp}$ to $800 \mathrm{bp}$. The prepared cDNAs were transformed into single-stranded template DNA (sstDNA) libraries by using the GS DNA Library Preparation kit (Roche Applied Science). sstDNA libraries were clonally amplified in a bead-immobilized form by using the GS emPCR kit (Roche Applied Science) and sequenced on the 454 Genome Sequencer FLX instrument.

\section{EST analyses and assembly}

The 454 sequencing reads were filtered by an in-house developed program to remove 5 ' adaptor sequences and low quality reads. The qualified reads were then assembled by CAP3 [50] using default parameters. Two kinds of assembly method were used: (1) assembly of the viruliferous and naïve read libraries, separately; (2) combining the viruliferous and naïve reads and assembly of all reads as one library. A cluster containing $\geq 2$ ESTs was named as a contig and those containing only one sequence were termed as singletons. The contigs and singletons were generally referred to as unigenes.

\section{Gene annotation}

After assembly, the unigene sequences (contigs and singletons) were subjected to BlastX similarity search against NCBI-NR non-redundant protein database. The parameters were set to: amino acid substitution matrix BLOSUM62, a statistical significance threshold of 10 for similarity matches, and costs to open an alignment gap and extend a gap of 11 and 1, respectively. Furthermore, to compare our results with other arthropods, additional searches (BlastX) were also performed against putative proteomes of 11 arthropod species whose genomes are in draft or completed, including Acyrthosiphon pisum, Aedes aegypti, Anopheles gambiae, Apis mellifera, Bombyx mori, Culex pipiens, Drosophila melanogaster, Ixodes scapularis, Nasonia vitripennis, Pediculus humanus, and Tribolium castaneum. For prediction of the secondary structure of proteins, the pfam database was searched using HMMER 2.0 program. For Gene Ontology (GO) analysis, outputs of the results from BlastX search against Swiss-Prot/TrEMBL databases (e-value $\leq 10^{-3}$ ) were combined. Non-redundant matches were then functionally annotated with GO terminology using Go-Diff [51]. For identification of microsatellite markers that can be used in genetic mapping and population genetics, SciRoKo v3.4 software was used to search 41,492 unigenes generated from the combined EST library. Searches were carried out to identify both perfect and interrupted $(\leq 2 \mathrm{bp}$ mismatch) motifs, with a minimum number of repeat unit of six.

\section{Expression profiling by semi-quantitative RT-PCR}

To verify the results of pyrosequencing of the ESTs of $L$. striatellus, we selected a total of 50 unigenes for RTPCR profiling. Total RNAs from viruliferous and naïve samples were treated with RNase-free DNase I (TaKaRa), and about $2 \mu \mathrm{g}$ of total RNA of each sample was reverse-transcribed by SuperScript ${ }^{\mathrm{TM}}$ III Reverse Transcriptase (Invitrogen) using oligo(dT) as primer. The PCR was carried out using the following thermal cycling profile: $95^{\circ} \mathrm{C}$ for $4 \mathrm{~min}$, followed by 20-25 cycles of amplification $\left(95^{\circ} \mathrm{C}\right.$ for $30 \mathrm{sec}, 55^{\circ} \mathrm{C}$ for $30 \mathrm{sec}$, and $72^{\circ} \mathrm{C}$ for $45 \mathrm{sec}$ ), and $72^{\circ} \mathrm{C}$ for $10 \mathrm{~min}$. Primers were designed based on contig sequences generated from ESTs of the same cluster (Additional file 6). The PCR products and their sizes were examined using $1 \%$ agarose gel electrophoresis followed by ethidium bromide staining. The elongation factor 2 (EF2) gene of L. striatellus was amplified as an endogenous loading control for testing the validity of template preparation. The expression of each gene was confirmed in at least two rounds of independent $\mathrm{RT}$-PCR reactions. 


\section{Additional material}

Additional file 1 Distribution of read lengths of viruliferous and naïve Laodelphax striatellus EST libraries. The figure provides statistics of read lengths of L. striatellus ESTs sequencing by the Roche 454-FLX platform. Additional file $\mathbf{2}$ List of Wolbachia genes identified in L. striatellus EST libraries. This table provides a list of genes from endosymbiotic bacteria Wolbachia that are expressed in cells of $\mathrm{L}$. striatellus.

Additional file 3 List of possible fungal genes identified in L. striatellus EST libraries. The table provides a list of genes that possibly expressed by endosymbiotic fungi, including yeast-like symbiotes.

Additional file $4 \mathrm{~L}$. striatellus genes abundantly transcribed in the viruliferous sample. The table provides a list of top 30 genes that highly expressed in the sample of viruliferous insects.

Additional file $\mathbf{5}$ List of genes selected for RT-PCR assay. This table shows the general feature of genes selected for RT-PCR analysis. Additional file 6 Sequences of primers used in RT-PCR profiling

\section{Authors' contributions}

FZ performed most of the analyses of the EST database and conducted the RT$P C R$ profiling. $H G$ and $T Z$ bred the insects and took part in extracting total RNA. $\mathrm{HZ}$ conducted 454 pyrosequencing, contig assembly and bioinformatic analysis. YZ provided the insect lines used in this study. WQ conducted the data analyses and prepared the manuscript. SW, RF, WQ and XC conceived, designed and coordinated the study. All authors have read, commented upon, and approved the final manuscript.

\section{Acknowledgements}

The authors thank two anonymous reviewers for their valuable comments on the manuscript. This work was supported by the Special Fund for Commonweal Industry (Nyhyzx07051) from the Department of Agriculture, P. R. China.

\section{Author Details}

1State Key Laboratory of Plant Genomics, Institute of Microbiology, Chinese Academy of Sciences, Beijing 100101, China, 2National Plant Gene Research Center, Beijing 100101, China, ${ }^{3}$ Graduate School of Chinese Academy of Sciences, Beijing 100039, China, 4 Shanghai-MOST Key Laboratory of Health and Disease Genomics, Chinese National Human Genome Center at Shanghai, Shanghai, 200032, China and 5Jiangsu Academy of Agricultural Sciences, Nanjing 210014, China

Received: 10 February 2010 Accepted: 13 May 2010

Published: 13 May 2010

\section{References}

1. Hogenhout SA, Ammar el D, Whitfield AE, Redinbaugh MG: Insect vector interactions with persistently transmitted viruses. Annu Rev Phytopathol 2008, 46:327-359.

2. Hohn T: Plant virus transmission from the insect point of view. Proc Natl Acad Sci USA 2007, 104(46):17905-17906

3. $\mathrm{Ng} \mathrm{JC}$, Falk BW: Virus-vector interactions mediating nonpersistent and semipersistent transmission of plant viruses. Annu Rev Phytopathol 2006, 44:183-212.

4. Toriyama S: Rice stripe virus: prototype of a new group of viruses that replicate in plants and insects. Microbio/ Sci 1986, 3(11):347-351.

5. Ramirez BC, Haenni AL: Molecular biology of tenuiviruses, a remarkable group of plant viruses. J Gen Virol 1994, 75(Pt 3):467-475.

6. Liang D, Qu Z, Ma X, Hull R: Detection and localization of Rice stripe virus gene products in vivo. Virus Genes 2005, 31(2):211-221.

7. Wu M, Sun LV, Vamathevan J, Riegler M, Deboy R, Brownlie JC, McGraw EA, Martin W, Esser C, Ahmadinejad N, et al.: Phylogenomics of the reproductive parasite Wolbachia pipientis wMel: a streamlined genome overrun by mobile genetic elements. PLOS Biol 2004, 2(3):E69.

8. Yassine $\mathrm{H}$, Osta MA: Anopheles gambiae innate immunity. Cell Microbio 2009, 12(1):1-9.

9. Lemaitre B, Hoffmann J: The host defense of Drosophila melanogaster. Annu Rev Immunol 2007, 25:697-743.

10. Brennan CA, Anderson KV: Drosophila: the genetics of innate immune recognition and response. Annu Rev Immunol 2004, 22:457-483.
11. Ashburner M, Ball CA, Blake JA, Botstein D, Butler H, Cherry JM, Davis AP Dolinski K, Dwight SS, Eppig JT, et al.: Gene ontology: tool for the unification of biology. The Gene Ontology Consortium. Nat Genet 2000, 25(1):25-29.

12. Consortium IAG: Genome sequence of the pea aphid Acyrthosiphon pisum. PLOS Biol 2010, 8(2):e1000313.

13. Li YC, Korol AB, Fahima T, Beiles A, Nevo E: Microsatellites: genomic distribution, putative functions and mutational mechanisms: a review. Mol Ecol 2002, 11(12):2453-2465.

14. Kofler R, Schlotterer C, Lelley T: SciRoKo: a new tool for whole genome microsatellite search and investigation. Bioinformatics 2007 23(13):1683-1685

15. Toriyama S, Takahashi M, Sano Y, Shimizu T, Ishihama A: Nucleotide sequence of RNA 1, the largest genomic segment of rice stripe virus, the prototype of the tenuiviruses. J Gen Virol 1994, 75(Pt 12):3569-3579.

16. Xiong $R$, Wu J, Zhou $Y$, Zhou $X$ : Identification of a movement protein of the tenuivirus rice stripe virus. J Virol 2008, 82(24):12304-12311.

17. Xiong R, Wu J, Zhou Y, Zhou X: Characterization and subcellular localization of an RNA silencing suppressor encoded by Rice stripe tenuivirus. Virology 2009, 387(1):29-40.

18. Saridaki A, Bourtzis K: Wolbachia: more than just a bug in insects genitals. Curr Opin Microbiol 2010, 13(1):67-72

19. Hedges LM, Brownlie JC, O'Neill SL, Johnson KN: Wolbachia and virus protection in insects. Science 2008, 322(5902):702.

20. Teixeira L, Ferreira A, Ashburner M: The bacterial symbiont Wolbachia induces resistance to RNA viral infections in Drosophila melanogaster. PLOS Biol 2008, 6(12):e2.

21. Kang L, Ma X, Cai L, Liao S, Sun L, Zhu H, Chen X, Shen D, Zhao S, Li C: Superinfection of Laodelphax striatellus with Wolbachia from Drosophila simulans. Heredity 2003, 90(1):71-76.

22. Fenn K, Blaxter M: Wolbachia genomes: revealing the biology of parasitism and mutualism. Trends Parasito/ 2006, 22(2):60-65.

23. Nikaido $\mathrm{H}$, Takatsuka Y: Mechanisms of RND multidrug efflux pumps. Biochim Biophys Acta 2009, 1794(5):769-781.

24. Tsilibaris V, Maenhaut-Michel G, Van Melderen L: Biological roles of the Lon ATP-dependent protease. Res Microbiol 2006, 157(8):701-713.

25. Suh SO, Noda H, Blackwell M: Insect symbiosis: derivation of yeast-like endosymbionts within an entomopathogenic filamentous lineage. Mol Biol Evol 2001, 18(6):995-1000.

26. Noda H, Nakashima N, Koizumi M: Phylogenetic position of yeast-like symbiotes of rice planthoppers based on partial $18 \mathrm{~S}$ rDNA sequences. Insect Biochem Mol Biol 1995, 25(5):639-646.

27. Noda H, Koizumi Y: Sterol biosynthesis by symbiotes: cytochrome P450 sterol C-22 desaturase genes from yeastlike symbiotes of rice planthoppers and anobiid beetles. Insect Biochem Mol Biol 2003, 33(6):649-658

28. Wang XH, Aliyari R, Li WX, Li HW, Kim K, Carthew R, Atkinson P, Ding SW: RNA interference directs innate immunity against viruses in adult Drosophila. Science 2006, 312(5772):452-454.

29. Kemp C, Imler JL: Antiviral immunity in drosophila. Curr Opin Immunol 2009, 21(1):3-9.

30. Dostert C, Jouanguy E, Irving P, Troxler L, Galiana-Arnoux D, Hetru C, Hoffmann JA, Imler JL: The Jak-STAT signaling pathway is required but not sufficient for the antiviral response of drosophila. Nat Immunol 2005, 6(9):946-953.

31. Carpenter J, Hutter S, Baines JF, Roller J, Saminadin-Peter SS, Parsch J, Jiggins FM: The transcriptional response of Drosophila melanogaster to infection with the sigma virus (Rhabdoviridae). PLoS One 2009, 4(8):e6838.

32. Arbouzova NI, Zeidler MP: JAK/STAT signalling in Drosophila : insight into conserved regulatory and cellular functions. Development 2006 133(14):2605-2616.

33. Agaisse $\mathrm{H}$, Perrimon $\mathrm{N}$ : The roles of JAK/STAT signaling in Drosophila immune responses. Immunol Rev 2004, 198:72-82.

34. Hedges LM, Johnson KN: Induction of host defence responses by Drosophila C virus. J Gen Virol 2008, 89(Pt 6):1497-1501.

35. Kaneko T, Silverman N: Bacterial recognition and signalling by the Drosophila IMD pathway. Cell Microbiol 2005, 7(4):461-469.

36. Kaneko T, Yano T, Aggarwal K, Lim JH, Ueda K, Oshima Y, Peach C, ErturkHasdemir D, Goldman WE, Oh BH, et al.: PGRP-LC and PGRP-LE have essential yet distinct functions in the drosophila immune response to monomeric DAP-type peptidoglycan. Nat Immuno/ 2006, 7(7):715-723. 
37. Kaneko T, Golenbock D, Silverman N: Peptidoglycan recognition by the Drosophila Imd pathway. J Endotoxin Res 2005, 11(6):383-389.

38. Costa A, Jan E, Sarnow P, Schneider D: The Imd pathway is involved in antiviral immune responses in Drosophila. PLoS One 2009, 4(10):e7436.

39. Avadhanula V, Weasner BP, Hardy GG, Kumar JP, Hardy RW: A novel system for the launch of alphavirus RNA synthesis reveals a role for the Imd pathway in arthropod antiviral response. PLoS Pathog 2009, 5(9):e1000582.

40. Watts TH: TNF/TNFR family members in costimulation of T cell responses. Annu Rev Immunol 2005, 23:23-68.

41. Gerardo NM, Altincicek B, Anselme C, Atamian H, Barribeau SM, de Vos M, Duncan E, Evans JD, Gabaldon T, Ghanim M, et al.: Immunity and other defenses in pea aphids, Acyrthosiphon pisum. Genome Biol 11(2):R21.

42. Tufail M, Takeda M: Molecular characteristics of insect vitellogenins. $J$ Insect Physiol 2008, 54(12):1447-1458.

43. Leblanc P, Desset S, Giorgi F, Taddei AR, Fausto AM, Mazzini M, Dastugue $B$, Vaury C: Life cycle of an endogenous retrovirus, ZAM, in Drosophila melanogaster. J Virol 2000, 74(22):10658-10669.

44. Brasset E, Taddei AR, Arnaud F, Faye B, Fausto AM, Mazzini M, Giorgi F, Vaury C: Viral particles of the endogenous retrovirus ZAM from Drosophila melanogaster use a pre-existing endosome/exosome pathway for transfer to the oocyte. Retrovirology 2006, 3:25.

45. Wan F, Anderson DE, Barnitz RA, Snow A, Bidere N, Zheng L, Hegde V, Lam LT, Staudt LM, Levens D, et al.: Ribosomal protein S3: a KH domain subunit in NF-kappaB complexes that mediates selective gene regulation. Cell 2007, 131(5):927-939.

46. Drakesmith $\mathrm{H}$, Prentice A: Viral infection and iron metabolism. Nat Rev Microbiol 2008, 6(7):541-552.

47. Drakesmith H, Chen N, Ledermann H, Screaton G, Townsend A, Xu XN: HIV-1 Nef down-regulates the hemochromatosis protein HFE, manipulating cellular iron homeostasis. Proc Natl Acad Sci USA 2005, 102(31):11017-11022.

48. Romeo AM, Christen L, Niles EG, Kosman DJ: Intracellular chelation of iron by bipyridyl inhibits DNA virus replication: ribonucleotide reductase maturation as a probe of intracellular iron pools. $\mathrm{J}$ Biol Chem 2001, 276(26):24301-24308.

49. Ng P, Wei CL, Sung WK, Chiu KP, Lipovich L, Ang CC, Gupta S, Shahab A, Ridwan A, Wong CH, et al.: Gene identification signature (GIS) analysis for transcriptome characterization and genome annotation. Nat Methods 2005, 2(2):105-111.

50. Huang X, Madan A: CAP3: A DNA sequence assembly program. Genome Res 1999, 9(9):868-877.

51. Chen Z, Wang W, Ling XB, Liu JJ, Chen L: GO-Diff: mining functional differentiation between EST-based transcriptomes. BMC Bioinformatics 2006, 7:72.

doi: $10.1186 / 1471-2164-11-303$

Cite this article as: Zhang et al., Massively parallel pyrosequencing-based transcriptome analyses of small brown planthopper (Laodelphax striatellus), a vector insect transmitting rice stripe virus (RSV) BMC Genomics 2010, 11:303

\section{Submit your next manuscript to BioMed Centra} and take full advantage of:

- Convenient online submission

- Thorough peer review

- No space constraints or color figure charges

- Immediate publication on acceptance

- Inclusion in PubMed, CAS, Scopus and Google Scholar

- Research which is freely available for redistribution

Submit your manuscript at www.biomedcentral.com/submit
C Biomed Central 